\title{
Flavia Solieri
}

\section{La nascita}

\section{della Repubblica Popolare Cinese Alcune priorità e linee di politica estera}

doi: 10.7358/ling-2014-002-soli

flavia.solieri@uniurb.it

Come è noto, la Repubblica Popolare Cinese（中华人民共和国 Zhonghua renmin gongheguo, RPC) venne fondata il primo ottobre del 1949.

Naturalmente, la data fondativa mantiene nella sua puntualità un'alta $\mathrm{e}$ sentita valenza simbolica, di spartiacque fra scomparsa della "vecchia Cina" ed emergere di una "nuova Cina". Allo stesso tempo, analisi e valorizzazione critica in particolare di fonti cinesi pubblicate tra la fine degli anni Ottanta e i primi anni Novanta permettono di delineare riguardo a quel passaggio storico un quadro di grande interesse e complessità: problemi e tentativi di risposta, continuità e discontinuità attraversano l'ottobre 1949, affondano nel passato e si proiettano verso il futuro, muovendo da un presente quanto mai arduo, carico di incognite e gravido di difficoltà. Dinamiche e processi relativi a quegli anni, così densi e determinanti, presentano quindi notevoli potenzialità di ricerca, sia in ambito interno sia in ambito internazionale.

Si cercherà in questo contributo di tratteggiare, in modo estremamente sintetico, alcune delle linee politiche e di azione concreta che agli albori della fondazione della RPC vennero individuate e portate avanti in politica estera. Non è un compito facile: nel senso che, come si accennerà, la nuova disponibilità negli ultimi decenni di fonti russe e cinesi ha portato a lavori in alcuni casi molto dettagliati, ma non univoci. Approcci di ampio respiro, tuttavia, proprio a fronte della pletora di fonti e interpretazioni disponibili e di una specializzazione sempre più marcata, conservano a maggior ragione la loro utilità - anche se devono accompagnarsi a una nota di modestia, e alla consapevolezza che quella che si propone è una delle sintesi possibili.

In modo particolare, un approccio che proponga una overview appare utile in relazione ad aspetti di politica estera: ambito in cui, come si vedrà, 
alcune grandi linee di fondo allora impostate da parte cinese non hanno perso la loro attualità, e dove un approccio specializzato è importante.

\section{IL CONTESTO INTERNAZIONALE}

\subsection{Washington e Pechino}

Il 30 giugno 1949 Mao Zedong (毛泽东) rese nota una linea di politica estera sintetizzata nella necessità di "pendere da una parte" (一边倒 yibiandao): in un mondo rigidamente bipolare, la futura Repubblica Popolare Cinese non avrebbe potuto non collocarsi nel campo dell'Unione Sovietica. La recente e più autorevole storiografia cinese riconduce quella decisione alle proprie radici autoctone: tale chiara scelta di campo sarebbe stata motivata cioè non tanto e non solo dalla indisponibilità americana, ma dall'avvicinarsi della convocazione della Conferenza politica consultiva（中国人民政治协商会议 Zhongguo renmin zhengzhi xieshang buiyi), l'organismo che avrebbe svolto le funzioni di conferenza rappresentativa nazionale (Solieri 2006, 129-31). Con la vittoria nella guerra civile e l'approssimarsi quindi di fondamentali passaggi fondativi e istituzionali, diventava imperativo per il Partito Comunista Cinese (中国共产党 Zhongguo Gongchandang, PCC) definire ed esplicitare i principi di base cui il nuovo Paese si sarebbe richiamato, in politica estera e interna: allo scopo anche di venire riconosciuto dall'Urss, ricevendone il sostegno necessario ad avviare recupero economico e ricostruzione, e un importante supporto alla sicurezza del nuovo Stato ${ }^{1}$. La scelta di campo cinese viene quindi letta muovendo da obiettivi propri e autodeterminati, e non come imposta dall'esterno o subita passivamente. Anche la sua collocazione temporale viene ricondotta al quadro cinese di allora: la grande avanzata militare in pieno svolgimento richiedeva infatti indirizzi chiari, che a loro volta sarebbero risultati particolarmente autorevoli proprio perché proposti in quel contesto di indiscutibili successi (Bo 1991, 1: 38-9).

Dunque, recentemente vengono sottolineate in Cina le ragioni domestiche per cui, tra i dirigenti del PCC, nell'estate del 1949 sarebbe in modo autonomo maturata la convinzione della vantaggiosità di una chiara presa di posizione a fianco dell'Unione Sovietica.

Quella decisione venne viceversa letta allora a Washington come un tra-

1 Tutti questi obiettivi erano variamente collegati ad aspetti di rilevanza interna: le interfacce, per così dire, interna ed esterna della situazione non sembrano separabili. 
dimento del "Chinese heritage", e dimostrazione pubblica di "subservience to a foreign power, Russia" (van Slyke, ed., 1967, xvi). Come si vedrà, la tendenza da parte statunitense a percepire Pechino come succube dell'Urss e priva di proprie prospettiva e autonomia decisionali sarà nel periodo qui considerato vivissima e costante - nonostante la disponibilità cinese ad aprire, a certe condizioni, canali di interazione con Washington. Allora, nel 1949, il tentativo di placare negli Stati Uniti l'opinione pubblica e quegli ambienti politici che, a fronte degli sviluppi in Cina, accusavano di incapacità e inconcludenza l'amministrazione Truman, comportò la pubblicazione di lì a poche settimane del ponderoso documento United States Relations with China, noto in genere come China White Paper. Esso rendeva noto tutto quanto gli Stati Uniti avevano fatto per sostenere in Cina il Partito nazionalista (国民党 Guomindang, Gmd), l'avversario diretto contro cui i comunisti stavano combattendo e prevalendo. La pubblicazione Paper, è interessante osservare, negli Stati Uniti non placò le critiche, e in Cina chiuse definitivamente ogni possibilità di avvicinamento tra il nuovo governo che di lì a poco si sarebbe formato e quello americano. Precluse anche la possibilità che i comunisti cinesi si differenziassero a breve dal Cremlino, contribuendo a rendere la questione manichea: "either friendship with the evil Soviet empire or friendship with democratic America" (Chang 1990, 37).

Negli Stati Uniti non solo il China White Paper non mise a tacere le critiche rivolte alla politica cinese del governo americano, ma anzi nei mesi successivi il clima di frustrazione e rabbia si intensificò: le accuse di inconcludenza si saldarono al sospetto che all'interno dell'amministrazione Truman, come in precedenza con Roosvelt, esistesse una cospirazione per sostenere i comunisti cinesi. Nacque così un'alleanza tra il senatore Joseph McCarthy, dell'ala conservatrice del Partito repubblicano, e il cosiddetto China Bloc, un gruppo di membri del Congresso poco interessati all'Europa e favorevoli piuttosto a una politica di massimo sostegno a Chiang Kai-shek (蒋介石, Jiang Jieshi). Il test atomico sovietico del settembre 1949 e alcuni segni di spionaggio a favore di Mosca parvero fornire una parziale spiegazione del male oscuro statunitense, di cui sia la "perdita della Cina" sia i rapidi progressi scientifici sovietici sembravano prove.

Fu su tale sfondo cupo e teso che venne fondata la Repubblica Popolare Cinese: come si vedrà, già dagli inizi del 1949 la dirigenza del PCC aveva da parte sua elaborato linee di politica estera importanti, e significativamente tra l'altro fortemente protettive.

Nonostante gli Stati Uniti continuassero a sostenere il Guomindang nella guerra civile, tra la primavera e l'estate del 1949 i comunisti cinesi tentarono di veicolare a Washington disponibilità e interesse per rapporti di tipo economico: mentre cioè il perdurare dell'impegno Usa a fianco dei nazionalisti preclu- 
deva la possibilità di instaurare relazioni diplomatiche, era aperta la possibilità di stringere tra i due Paesi, a certe condizioni, accordi di natura commerciale. Nella primavera-estate del 1949 Huang Hua (黄华) ${ }^{2}$, nel corso di incontri a Nanchino con John Leighton Stuart, ambasciatore americano in Cina, espresse l'interesse del PCC per un riconoscimento statunitense del proprio governo, sottolineando la necessità di avere relazioni, commerciali e non solo, con Paesi stranieri. Da parte sua, Stuart accennò che presupposti per un riconoscimento statunitense erano che la Cina rispettasse i trattati (senza specificare se trattati internazionali, o conclusi dal Guomindang, o entrambi) e che il popolo cinese appoggiasse chiaramente il nuovo governo; ribadì anche i timori di Washington a fronte della teoria comunista della rivoluzione mondiale attraverso la violenza, che il PCC avrebbe fatto propria (Shaw 1982, 81-2).

Linteresse allora segnalato da Huang era espressione di valutazioni che il Comitato centrale del PCC (中共中央 ZhongGong Zhongyang) aveva diffuso in febbraio, e che contemplavano la possibilità, al fine di sviluppare la economia nazionale della "nuova Cina" (新中国的国民经济 Xin Zhongguo de guomin jingji), di stringere accordi commerciali anche con Paesi con cui non erano ancora state avviate relazioni diplomatiche: condizioni erano la loro utilità per la Cina, che non pregiudicassero sovranità e indipendenza nazionali, che fossero sotto controllo governativo (Zhongyang dang'an bian 1992: 18: 138). Ma quello, come altri messaggi che tra la primavera e l'estate del 1949 i comunisti cinesi mandarono a Washington segnalando interesse per commercio, crediti e assistenza tecnica - e introducendo una sofisticata distinzione tra linea politica e rapporti di natura economica - non vennero colti. In particolare, in giugno Zhou Enlai (周恩来) informò O. Edmund Clubb, console statunitense a Pechino, dell'interesse a investimenti e operazioni commerciali, se di reciproco vantaggio (Shaw 1982, 85). L'intenzione di fare affari anche con Paesi capitalisti, a condizione che venissero rispettati sovranità nazionale e interessi del popolo cinese, emerge anche da un telegramma che Mao Zedong inviò da Mosca al Comitato centrale del PCC nel dicembre dello stesso anno (Solieri 2006, 182).

Nonostante il fortissimo orientamento bipolare della situazione politica mondiale, i comunisti cinesi auspicavano dunque, a patto che alcune condizioni di base venissero rispettate, contatti economici, tecnologici e formativi anche con Stati Uniti, Europa e Giappone. A condizione che Paesi politicamente ostili non tentassero di vanificare la vittoria del PCC, di fatto esisteva dunque da parte cinese una disponibilità alla cooperazione economica: le controparti del blocco gravitante attorno a Washington allora però non seppero, o più probabilmente non vollero, vederla. Nel clima politico dell'epoca, negli Stati Uniti si

2 Tutti questi obiettivi erano variamente collegati ad aspetti di rilevanza interna: le interfacce, per così dire, interna ed esterna della situazione non sembrano separabili. 
affermò una lettura della vittoria dei comunisti cinesi che non ne considerava specificità storica, culturale e politica, compresi i loro rapporti in passato più volte difficili con Mosca e il Comintern; li si percepì e descrisse come puppets nelle mani dell'Unione Sovietica ("Moscow Communists") e dei suoi piani di dominazione del mondo:

Whether or not the motives of the so-called liberal Chinese Communists in approaching our representatives are genuine, their actions are in complete harmony with traditional Communist tactics and are in no manner contradictory to Communist objectives of world-domination. Economic assistance to Moscow Communists can be justified only on the basis of strictly quid pro quo transactions wherein the benefits to the West are for the long term equal to or greater than the benefits to the Communist dictatorships. (National Archives, 1945-1949)

Frustrazione e risentimento derivanti da quella che negli Stati Uniti veniva definita "loss of China" ${ }^{3}$ si tradussero in attendismo e senso di impotenza, accecando anche nei confronti di quelle potenzialità che da parte cinese, come si è visto, si intendeva valorizzare. Si tradussero anche in una pletora di dichiarazioni confuse e contraddittorie ${ }^{4}$, da parte di questo o quell'esponente dell' $e$ stablishment, su temi per la Repubblica Popolare Cinese altamente sensibili: quali le intenzioni dell'amministrazione Truman circa il perimetro difensivo statunitense nel Pacifico o Taiwan (台湾), dove nel dicembre del 1949 era riparato anche Chiang Kai-shek.

Da quel momento, la semplice esistenza dell'isola come roccaforte anticomunista inibì fortemente un avvicinamento alla RPC, e il punto di vista di Acheson, per cui qualsiasi mossa statunitense a favore di Taiwan avrebbe stretto ancora di più i legami tra Mosca e Pechino, iniziò a indebolirsi. Pressioni per aiuti militari americani a Chiang iniziarono a essere avanzate tra gli altri dal generale Douglas MacArthur, e presto la situazione apparve confusa: mentre agli inizi del gennaio 1950 Truman affermava che al momento gli Stati Uniti non erano interessati a stabilire basi militari a Formosa, ambienti militari rendevano noto che riguardo all'isola tutte le opzioni restavano aperte (Stueck 1981, 142). Da parte loro, fonti della Repubblica Popolare Cinese rendevano noto che 250 carri armati statunitensi, e una trentina di consiglieri militari, erano già sbarcati

3 Huang Hua aveva frequentato a Pechino la Università Yanjing (燕京), di cui Stuart era stato Presidente; guidava allora l'Ufficio per i residenti stranieri della Commissione militare di controllo di Nanchino.

4 Tra le ragioni della inconsistenza della politica statunitense nei confronti del corso che gli eventi avevano preso in Cina, anche le diverse sensibilità al riguardo presenti ai vertici della politica statunitense: dal relativamente carente senso delle relazioni internazionali di Truman, l'asse portante della cui politica dal 1945 al 1950 rimase il containment, al relativo disinteresse per l'Asia del Segretario di Stato Dean Acheson, Atlantista quasi esclusivamente interessato a legare Giappone e Germania all'Occidente, al rigido obiettivo di containment nei confronti di Mosca di George F. Kennan. 
sull'isola (Gurtov, Hwang 1980, 41). Dal gennaio 1950, pesanti bombardamenti su città costiere cinesi vennero effettuati dai nazionalisti con bombardieri costruiti in America. Ma, sempre in gennaio, Dean Acheson affermava che Corea del Sud e Taiwan erano escluse dal perimetro difensivo statunitense: affermazione che sarebbe stata smentita di lì a pochi mesi dalle reazioni americane allo scoppio del conflitto nella penisola.

\subsubsection{Il documento NSC-68}

Nella capitale statunitense un salto di qualità nello percezione della RPC si ebbe con un documento elaborato tra febbraio e marzo 1950 da una commissione congiunta Stato-Difesa, poi trasmesso da Truman al National Security Council e da questo denominato NSC-68. NSC-68 trattava per la prima volta la minaccia sovietica come anzitutto militare, preconizzando la possibilità di una Pearl Harbor atomica da parte di Stalin contro gli Stati Uniti a partire dal 1954. In un contesto di quel tipo, qualsiasi affermazione comunista veniva vista come tassello di un piano volto alla conquista del mondo, in una inespressa equazione col Terzo Reich. Così, la vittoria del PCC nella guerra civile cinese sollevava comunque allarme, perché metteva in discussione la volontà americana di resistere al diffondersi del "Comunismo" su scala planetaria, e anche zone periferiche come la Corea o Taiwan diventavano importanti.

Il documento invocava dunque una politica di riarmo, sia atomico che convenzionale, sottintendendo che un forte aumento delle spese militari avrebbe favorito lo sviluppo e consolidato la leadership americana nel "mondo libero". A partire da quel momento l'espansione comunista avrebbe dovuto essere ostacolata anche attraverso dirette operazioni militari. Pur comportando un notevole riorientamento della politica statunitense, il documento NSC-68 venne considerato di massima segretezza; la nuova lettura che a Washington si faceva dello scenario mondiale non venne resa nota, e i segnali pubblici che raggiungevano Mosca e Pechino restarono immutati e contraddittori. Il 30 maggio 1950 Dean Rusk, nuovo assistente di Acheson per l'Estremo Oriente, raccomandò una missione a Taiwan, la cui caduta in mani comuniste veniva ritenuta imminente, del senatore John Foster Dulles; contemporaneamente, unità navali americane sarebbero state pronte a stringere un cordone sanitario attorno all'isola. Tuttavia, ancora il 23 giugno, un paio di giorni prima dello scoppio della guerra di Corea, Acheson ripeteva che gli Stati Uniti avrebbero continuato a non fornire materiale né assistenza militare a Chiang Kai-shek.

Nel periodo considerato, l'esistenza da parte statunitense, riguardo ad aspetti direttamente collegati alla sicurezza della Repubblica Popolare Cinese, di punti di vista niente affatto univoci e di affermazioni pubbliche in contrasto 
con la realtà dei fatti, accrebbe l'incertezza di Pechino circa le reali intenzioni di Washington. A complicare ulteriormente le cose, come si vedrà, anche il fatto che il seggio cinese al Consiglio di sicurezza delle Nazioni unite restasse attribuito alla Cina nazionalista.

\subsection{Mosca e Pechino}

Il 2 ottobre 1949 l'Unione Sovietica fu il primo Stato a riconoscere ufficialmente la Repubblica Popolare Cinese. L'ambasciatore russo che vi presentò - o più corretto sarebbe dire vi trasferì - le proprie credenziali era stato fino a quel momento accreditato presso Chiang Kai-shek: questa circostanza, apparentemente marginale, rimanda efficacemente ad altri elementi di complessità insiti nella situazione, oltre ai problemi esistenti con Washington.

Qui è solo il caso di ricordare brevemente come a Jalta nessuno dei tre Grandi avesse previsto una vittoria comunista in Cina; quanto concordato relativamente a quel Paese fu pochi mesi dopo incorporato in un trattato che nell'agosto del 1945 venne firmato a Mosca tra Unione Sovietica e governo cinese nazionalista, governo che veniva riconosciuto dall'Urss come unico legittimo rappresentante del popolo cinese ${ }^{5}$.

È stato sostenuto che la imprevista vittoria del PCC nella guerra civile abbia dischiuso per l'Urss il rischio di un corso politico suscettibile di entrare in collisione con i propri interessi, minacciandone il ruolo dominante nel movimento comunista mondiale (Medvedev 1986, 19); costituendo poi una variabile non prevista a Jalta, comportava anche la eventualità che Mosca venisse accusata di tradimento rispetto a quanto allora concordato: aspetto verso cui Stalin, come si vedrà anche in relazione alla evoluzione della situazione sul fronte coreano, mostrò grandi attenzione e prudenza.

Alla luce di queste considerazioni, il riconoscimento della Repubblica Popolare Cinese da parte dell'Unione Sovietica non deve apparire un fatto scontato. A prepararlo concorse anche una missione segreta di Liu Shaoqi (刘少奇) a Mosca nell'estate 1949; il primo contatto diretto tra un inviato di Stalin e i dirigenti del PCC era però già avvenuto nel gennaio 1949, a Xibaipo (西柏坡), quando la vittoria PCC nella guerra civile si delineava con chiarezza. Significativamente, si trattò anche in questo caso di un incontro segreto. L'inviato di Stalin, Anastas Mikojan, si limitò quasi esclusivamente ad ascoltare: è

5 Dichiarazioni eterogenee e non coordinate su temi di politica estera da parte di esponenti politici americani continuano a verificarsi e non cessano di essere fonte di irritazione per la Cina, per cui viceversa temi di politica estera risentono di una impostazione assolutamente coerente, decisa a livello di governo centrale. 
ipotizzabile che sull'incontro aleggiassero anche le ombre proiettate da diverse circostanze passate, in cui decisioni del Cremlino avevano avuto nei fatti conseguenze quanto mai gravi per i comunisti cinesi (Samarani 2004, 65-199).

Stalin non cessò di manifestare pubblicamente grande prudenza nei confronti dell'evolvere della situazione cinese. Si è già accennato a come fino all'ultimo l'ambasciatore sovietico avesse seguito Chiang Kai-shek; è il caso di accennare brevemente qui anche a come, verosimilmente nella primavera del 1949, prima dell'attraversamento dello Changjiang (长江, Yangzi), sarebbe stata avanzata da parte sovietica ai dirigenti del PCC la proposta di non procedere verso Sud e attestarsi a Nord del grande fiume - preludio a una divisione della Cina in un Nord controllato dal PCC e in un Sud dal Gmd (Solieri 2006, 183-4). Si tratta di un passaggio storico interessante: che la proposta sia stata avanzata apparirebbe in linea con la prudenza di Stalin ${ }^{6}$; che non sia stata raccolta rivela a sua volta il margine di autonomia, relativamente certo a quanto la situazione permetteva, con cui i comunisti cinesi potevano muoversi anche nei confronti di Mosca. Mentre Washington andava come ricordato sviluppando una concezione monolitica del "blocco comunista", circostanze come questa o come la relativa autonomia decisionale della Corea del Nord riguardo al tentativo di riunificate il Paese - dimostrano indubbiamente quanto, in relazione al periodo e allo scenario considerati, la percezione statunitense fosse riduttiva.

L'atteggiamento sovietico nei confronti della "nuova Cina" può dunque essere letto come dettato non solo dall'intento di ostacolare, o quantomeno non favorire, ritardare, l'emergere sulla scena internazionale di un altro grande Paese a guida comunista, ma anche da cautela nei confronti degli impegni assunti a Jalta, in modo particolare con gli Stati Uniti. Le due linee interpretative non si elidono a vicenda, e per lo più il corso delle relazioni sino-sovietiche del periodo è segnato da questa ambiguità.

Particolarmente interessante, si potrebbe dire esemplare, la vicenda del boicottaggio sovietico in seno alle Nazioni unite, in relazione proprio alla fondazione della Repubblica Popolare Cinese. Rimandando al paragrafo successivo una breve sintesi di come la Repubblica popolare si rapportò alla organizzazione internazionale, qui è sufficiente anticipare che ancora a metà gennaio 1950 le circostanze non erano particolarmente avverse all'ammissione di Pechino: anche volendo riferirsi al criterio che subordinava l'ammissione al riconoscimento, cinque degli undici membri del Consiglio di sicurezza avevano già riconosciuto la RPC (Unione Sovietica, India, Jugoslavia, Gran Bretagna, Norvegia), e si ritiene che altri due - Francia ed Egitto - fossero in procinto di farlo (Tang 1966, 524). L'atteggiamento americano sembrava improntato ad attendismo e cautela: nel senso che si aspettava probabilmente che la caduta di Taiwan, allora ritenuta a

6 In un contesto, è bene ricordare, ancora di monopolio nucleare statunitense. 
Washington imminente, eliminasse 'naturalmente' una imbarazzante scelta tra le due Cine, nel momento in cui gran parte dello schieramento politico e dell'opinione pubblica statunitense protestavano per un atteggiamento troppo accomodante nei confronti dei comunisti e di non chiaro sostegno a Chiang Kai-shek.

In quelle circostanze non facili, ma di non radicale ostilità di Washington verso Pechino, il 13 gennaio il rappresentante sovietico al Consiglio di sicurezza, Jacob Malik, presentò un progetto di risoluzione che prevedeva l'espulsione del rappresentante della Cina nazionalista ${ }^{7}$. Quando il progetto venne respinto, Malik iniziò in segno di protesta ad astenersi dal partecipare alle sedute del Consiglio. Il momento scelto sembrò subito non felice, essendo proprio allora il rappresentante del Guomindang a tenere la Presidenza, per cui sarebbe stato senz'altro più prudente aspettare il mese successivo, quando gli sarebbe subentrato il rappresentante di Cuba.

Lasciando qui sullo sfondo ulteriori elementi di complessità che nelle relazioni tra RPC e Usa si svilupparono nel corso del gennaio 1950, è stato osservato che allora il delegato sovietico in qualche modo 'protestò troppo': la Russia prese vigorosamente le parti di Pechino, gettò accuse a destra e manca, in poche parole sembrò darsi da fare per creare la massima opposizione alla causa della Repubblica popolare. Mosca si mosse all'interno di un'ottica bipolare del mondo, agganciando saldamente al proprio carro, per così dire, le rivendicazioni dei comunisti cinesi; tuttavia, così facendo, nella misura in cui un loro accoglimento sarebbe equivalso a una vittoria diplomatica sovietica, l'opposizione statunitense ne venne grandemente rafforzata. Il comportamento di Malik parve allora al Segretario generale delle Nazioni unite Trygve Lie basato su una calcolata politica di scoraggiare, piuttosto che incoraggiare, il riconoscimento della Repubblica Popolare Cinese (Lie 1954, 250). È in tal senso che l'operato del rappresentante sovietico può apparire come dettato in realtà dall'intento di rafforzare l'isolamento della Cina comunista, accentuandone la dipendenza da Mosca e riducendo al minimo il rischio di 'deviazioni'.

Nella prima metà del 1950 Pechino era dunque da un lato raggiunta da segnali confusi circa le intenzioni statunitensi relativamente a Taiwan; dall'altro, era praticamente priva di canali di comunicazione internazionali. Nel giugno, dei quattordici paesi non comunisti che avevano stabilito relazioni ufficiali con la RPC, cinque (Svezia, Danimarca, India, Birmania, Indonesia) avevano proceduto a uno scambio di ambasciatori. Con la Gran Bretagna i rapporti erano limitati a livello di incaricati d'affari, in segno di protesta per la perdurante attività consolare inglese a Taiwan. Con questa solitaria eccezione, Pechino non aveva relazioni con alcuna grande potenza, a eccezione dell'Unione Sovietica.

$\mathrm{E}$, anche in questo caso, quando il 25 giugno scoppiò il conflitto coreano,

7 Si accennerà nel paragrafo successivo alla questione della rappresentanza cinese in sede di Consiglio. 
Malik era assente dal Consiglio di sicurezza, continuandone il boicottaggio. Non fu quindi in condizione di porre il proprio veto alla proposta statunitense di un intervento internazionale nel conflitto: estrema misura di prudenza, con ogni verosimiglianza, affinché l'Unione Sovietica non venisse associata all'evolvere delle situazione estremo-orientale. In ogni caso, le conseguenze per la $\mathrm{RPC}$ furono gravi e durature.

\subsubsection{Il trattato del febbraio 1950}

L'Unione Sovietica fu riluttante anche a negoziare un trattato con la RPC, che rimpiazzò - cambiato l'interlocutore cinese - quello firmato a Mosca nell'agosto del 1945 con la Cina nazionalista.

Grazie in particolare alla disponibilità di materiale proveniente dagli archivi dell'ex-Unione Sovietica, la conoscenza delle trattative che precedettero il 14 febbraio 1950 la firma a Mosca del trattato di amicizia, alleanza e mutua assistenza tra i due Paesi - nonché di protocolli in precedenza segreti - ha fatto grandissimi passi avanti. Non è certamente questa la sede per entrare in dettaglio ${ }^{8}$; le differenze in enfasi e prospettiva che emergono tra due importanti commenti alla firma, uno di parte russa e l'altro di parte cinese, introducono comunque qualche spunto di riflessione: lo stesso 14 febbraio un editoriale della Pravda ricordava come la decisiva vittoria cinese fosse stata resa possibile dalla sconfitta del fascismo tedesco e dell'imperialismo giapponese, sconfitta in cui l'Unione Sovietica, guidata da Stalin, aveva avuto un ruolo determinante. Il giorno successivo Zhou Enlai affermava che la cooperazione tra i due Stati era stata resa possibile dalla vittoria del popolo cinese, sotto la guida del Presidente Mao Zedong (Simmons 1975, 73).

Il trattato, che nelle sue linee generali non si discostava molto da quello firmato cinque anni prima con la Cina nazionalista (Samarani 2004, 236-7), aveva una natura difensiva, per cui le due parti si impegnavano a sostenersi a vicenda in caso di attacco da parte del Giappone o di un Paese di questo alleato: circostanza importante a fronte in particolare del successivo intervento cinese in Corea. A differenza che nell'accordo col Guomindang del 1945, il trattato prevedeva in prospettiva la rinuncia ai diritti sovietici sul Nordest (东北 Dongbei), la vasta regione spesso ricordata sulla pubblicistica occidentale come Manciuria. L'Urss concesse poi alla Cina un prestito di 300 milioni di dollari al tasso di interesse dell' $1 \%$, in genere giudicato al di sotto delle aspettative cinesi (poco prima era stato concesso alla Polonia un prestito di 450 milioni di dollari senza interessi).

Per quanto, come sostenuto in particolare da Dieter Heinzig, gravide di ragioni di tensione e risentimento siano state le trattative che precedettero la

\footnotetext{
8 Heinzig (1998) riporta tra l'altro l'intero testo del trattato e degli altri accordi bilaterali.
} 
firma del trattato - così come non univoco il comportamento sovietico in relazione a nascita, visibilità e presenza internazionali della Repubblica Popolare Cinese - si può sostenere che l'accordo, pur con i suoi limiti, ha garantito la sopravvivenza della "nuova Cina". È un tipo di analisi che, in termini di estrema sintesi, appare difficilmente contestabile, qualora si pensi all'escalation che di lì a poco si avrà in Corea, e alla funzione di potente deterrente, nei confronti in particolare di un attacco nucleare alla $\mathrm{RPC}^{9}$, che il trattato avrà.

Per quanto l'intervento cinese a sostegno della Corea del Nord sia stato costoso in termini umani e non solo, e gravido di conseguenze nefaste; e per quanto duro sostenere per mesi i combattimenti prima che fosse fornita copertura aerea da parte russa, appare infatti assai verosimile che, nel clima dell'epoca, il coinvolgimento cinese nel conflitto avrebbe potuto avere conseguenze ancora più gravi se dietro a Pechino non ci fosse stata l'Unione Sovietica.

\section{LE RELAZIONI INTERNAZIONALI DELLA "NUOVA CINA"}

\subsection{Alcuni aspetti teorici}

Fu nel gennaio del 1949 che il Comitato centrale del PCC emise da Xibaipo un documento relativo al "lavoro nelle relazioni estere" (Zhongyang dang' an bian, 1992, 18: 44): nonostante la evoluzione militare non avesse ancora comportato l'attraversamento dello Changjiang e la proclamazione della nascita della Repubblica popolare (che avvenne a sua volta quando la riunificazione dell'intero Paese era militarmente incompiuta) fosse ancora lontana, in quella formulazione era interessantemente già implicita la dimensione, la prospettiva, di rapporti internazionali da Stato a Stato.

La "nuova Cina" non avrebbe subito passivamente situazioni imposte o ereditate dal passato, ma avrebbe mantenuto "l'iniziativa nelle proprie mani", muovendosi in base ad alcuni principi e priorità da essa stessa definiti, in cui fermezza e disponibilità si combinavano ed erano entrambe presenti (Solieri 2006,179). Esistevano disponibilità, ma non erano incondizionate; a suggerire di non avere fretta nello stabilire relazioni diplomatiche, anche la gravità della situazione interna, cui sarebbe spettata la priorità.

Nel febbraio 1949 alcune grandi linee guida, che orientarono i primi passi in terreno internazionale della RPC, vennero sintetizzate da Mao a Mikojan, inviato come ricordato da Stalin a Xibaipo per una prima presa di contatto, in:

9 Opzione più volte considerata da MacArthur, comandante della spedizione internazionale. 
“Mettere su un nuovo focolare" (另起炉灶 lingqi luzao) e “Invitare ospiti solo dopo aver fatto pulizia in casa” (打扫干净屋子再请客 dasao ganjing wuzi zai qing ke) (Shi 1991, 379-81).

Con "Mettere su un nuovo focolare" si intendeva che non sarebbero state riconosciute le relazioni diplomatiche avviate dal Partito nazionalista, ma si sarebbe ripartiti da basi del tutto nuove.

L'allacciare relazioni diplomatiche - questo il senso della seconda affermazione - sarebbe poi venuto in subordine rispetto al ripristino di condizioni di relativa normalità nel Paese. Nell'ultimo secolo la Cina era stata scossa da crisi interna, grandi rivolte, aggressione e penetrazione commerciale e finanziaria straniera, guerre, violenza endemica, fasi di guerra civile, inevitabilmente accompagnate tra l'altro da una lunga serie di fenomeni degenerativi e di crisi del tessuto sociale: dal banditismo alle società segrete, dalla prostituzione alla violenza endemica nelle campagne, al fenomeno del warlordismo, e in generale da un alto livello di militarizzazione. A queste circostanze, comunque di notevolissima gravità per chiunque volesse affrontare un compito di risanamento anche sociale, si aggiungeva la presenza di centinaia di migliaia di soldati sbandati e irriducibili del Gmd, e di reti di spionaggio facenti capo al Partito nazionalista o a paesi stranieri: agenti la cui attività in Cina risaliva al patto antiComintern stretto da Germania, Giappone e Italia; agenti di Taiwan; agenti assoldati, a diverso titolo, da agenzie di intelligence statunitensi.

Se dunque nel senso più ampio il "fare pulizia in casa", usando la metafora di cui sopra, comprendeva evidenti priorità di tipo economico-finanziario, produttivo, logistico, organizzativo e una pletora di compiti di natura sociale, complessità e pericolosità del tutto particolari erano presentate dalla presenza, per lo più sotterranea, di forze ostili al nuovo governo, a volte guidate e finanziate dall'estero: terminali operativi occulti alle dipendenze di paesi stranieri e forze nemiche ${ }^{10}$.

Era poi imprescindibile rifiutare tutti i "trucchi dell'imperialismo", cioè i tentativi allora messi in campo dall'esterno per intervenire nell'andamento della guerra civile: non permettendo ad alcun Paese né all'ONU di mediarvi o avervi un ruolo. Delineandosi agli inizi del 1949 una inattesa vittoria del PCC, era necessario rifiutare proposte straniere di mediazione e interferenze negli affari politici interni (内政 neizheng) ${ }^{11}$.

10 Dati che emergono da fonti d'archivio, o anche memorie di protagonisti, inducono a non sottostimare in alcun modo il fenomeno. Qui si può indicativamente accennare a come ancora un anno dopo, agli inizi del 1950, fossero militarmente attivi circa 500.000 uomini del Partito nazionalista, e, finanziate dagli Stati Uniti attraverso Hong Kong e Macao, fossero operanti organizzazioni quali il Sino-American Information Bureau, il South-East Asia Information Bureau, la Sino-American Co-operation Organization, facenti capo agli Stati Uniti e al Partito nazionalista cinese.

11 Mao Zedong in quello stesso periodo denunciava le diverse proposte internazionali di mediazione 
Proprio in tal senso Mao Zedong corresse nel maggio dello stesso anno, quando erano in corso a Nanchino i ricordati contatti a tra Huang Hua e Leighton Stuart, un documento elaborato dal locale comitato di partito (市委 shiwei, nella forma abbreviata):

Nessun Paese straniero deve interferire nella politica interna cinese. Deve finire quel tipo di politica per cui in passato gli Stati Uniti, aiutando il Guomindang nella guerra civile, hanno interferito nella politica interna cinese. Se il governo americano desidera provare a costruire relazioni diplomatiche con noi, deve cessare tutte le attività di sostegno al Guomindang, e rompere i rapporti con ciò che resta delle sue forze reazionarie. Questo non significa pretendere genericamente che il governo americano faccia ancora di più per il popolo cinese. Affermando questo, è possibile che diate agli americani l'impressione che anche il PCC speri in un aiuto americano.

Adesso si deve pretendere che gli Stati Uniti smettano di aiutare il Guomindang, che rompano i rapporti con quel che resta delle sue forze reazionarie, che non interferiscano mai in problemi di politica interna cinese. Non si deve chiedere che l'America faccia qualcosa «di utile alla causa del popolo cinese». Usando questa formulazione, sembrerebbe che il governo americano abbia già fatto qualcosa di utile per la causa del popolo cinese, ma abbia fatto un po' poco, e che ci sia bisogno di chiedere che faccia "ancora di più". Perciò non è appropriata. (ZhongGong zhongyang wenxian yanjiushi bian 1993, 3: 499)

In politica estera uno dei grandi orientamenti di fondo era dunque di operare una discontuinità radicale col passato in cui la Cina aveva subito l'imperialismo delle potenze straniere, e col loro framework concettuale delle "international obligations". Allo stesso tempo, i dirigenti di Pechino intendevano che con la "nuova Cina" un soggetto politico nuovo entrasse a pieno titolo nella comunità internazionale.

Da subito, i comunisti cinesi avevano manifestato un vivo interesse per l'Organizzazione delle nazioni unite, come testimonia la partecipazione di un loro rappresentante alla conferenza costitutiva a San Francisco nell'aprile 1945. Il Programma comune (共同纲领 Gongtong gangling), una forma di Costituzione provvisoria adottata il 29 settembre 1949, affermava la volontà della Repubblica Popolare Cinese di prendere il proprio posto nel campo della pace e democrazia internazionali, "per opporsi alla aggressione imperialista

che venivano da più parti avanzate; i nazionalisti tentarono di coinvolgervi anche l'Unione Sovietica. La non interferenza negli affari interni cinesi da parte di entità straniere - che costituì e costituisce un altro importante orientamento di fondo della politica estera della RPC - era già presente in un documento del febbraio 1948. Vi si sosteneva che condizione perché i “residenti stranieri” (外国侨民 waiguo qiaomin) potessero continuare a risiedere nelle zone controllate dai comunisti era che non danneggiassero la "sovranità del nostro Paese" (我国家主权 wo guojia zhuquan), naturalmente in primo luogo non sostenendo attività di sabotaggio da parte di soldati nazionalisti o mettendo a repentaglio il governo democratico (Zhongyang dang'an bian 1992, 17:35). 
e difendere una durevole pace mondiale" ${ }^{12}$. Relativamente al problema della ammissione all'ONU, la entità "Cina" ne è membro fin dall'origine: il problema che si poneva non era se ammettere Pechino quale nuova entità sovrana che solo recentemente aveva acquistato una personalità legale internazionale, ma se riconoscere questa o Taibei come governo "cinese".

Nel suo telegramma del 15 novembre 1949 a Trygve Lie, Segretario generale dell'ONU, il ministro degli Esteri Zhou Enlai, in rappresentanza del governo centrale della Repubblica Popolare Cinese, impostava correttamente il problema, limitandosi a chiedere che venisse revocato alla delegazione del governo nazionalista cinese il diritto di rappresentare il popolo cinese presso le Nazioni unite: una volta preso atto che la maggioranza del popolo cinese si riconosceva nel governo di Pechino, il subentro di quest'ultima sarebbe stato automatico. Tanto più che la conferenza del Cairo aveva stabilito nel 1943 che dopo la fine della guerra col Giappone Taiwan, colonia giapponese dal 1895, sarebbe tornata alla Cina. Il telegramma di Zhou venne reso noto solo dopo una settimana, dietro insistenza della delegazione polacca. Il giorno successivo, la delegazione sovietica protestò per la presenza dei nazionalisti e annunciò che Mosca non li riconosceva più come rappresentanti della Cina alle Nazioni unite. Il presidente dell'assemblea plenaria ritenne i tempi non maturi per discutere del problema, in conseguenza del fatto che dei cinquanta Stati membri solo quattro (Unione Sovietica, Polonia, Cecoslovacchia e Jugoslavia) avevano riconosciuto la Repubblica popolare. In questo modo venne creata ad hoc la dottrina per cui l'ammissione di uno Stato all'ONU era subordinata al riconoscimento di quello stesso Stato da parte della maggioranza dei Paesi membri: dottrina che non ha fondamento dal punto di vista del diritto internazionale ed è stata poi abbandonata, ma che allora ebbe il risultato di subordinare l'ingresso della Repubblica popolare al placet degli Stati Uniti, che potevano nei fatti controllare la maggioranza dei voti all'assemblea plenaria, e in sede di Consiglio di sicurezza disporre del potere di veto.

\subsection{Modalità operative sul campo}

Si è visto brevemente come alcune linee fondamentali che orientarono l'approccio alla politica estera nei primissimi tempi della Repubblica popolare vennero formulate nel gennaio del 1949.

Significativamente, l'Esercito popolare di liberazione (人民解放军 renmin jiefangjun, Epl) stava per conquistare Pechino (北京 Beijing) ${ }^{13}$ e Tianjin (天津):

12 Significativamente, è un aspetto sottolineato già in un volume - Whiting1960 - che, relativamente all' intervento cinese in Corea, non è recente, ma conserva l'autorevolezza e il valore di un classico.

13 Dal 1928 il governo nazionalista di Nanchino aveva ribattezzato Beijing ("capitale del Nord") in 
l'ingresso nelle due metropoli avrebbe dal punto di vista militare significato la conclusione di una delle tre grandi campagne che tra la fine del 1948 e gli inizi del 1949 segnarono le sorti del conflitto, comportando il controllo della Cina del Nord; dal punto di vista concreto, posero l'Epl di fronte ad aspetti di notevolissima complessità gestionale. L'ingresso in quelle grandi città comportò tra l'altro l'impatto concreto con un notevole numero di stranieri, tra cui anche diplomatici - e con attività di ogni tipo: scuole, imprese, ospedali, agenzie di stampa, chiese, banche, ecc., da loro gestite. Fu perciò allora che si dovette per così dire portare avanti sul campo, trasferendole nel contesto di grandi città e traducendole al contempo in modalità operative concrete verso gli stranieri in Cina, le linee politiche di fondo che nei confronti dei Paesi stranieri erano state appena impostate ${ }^{14}$. Il problema di una corrispondenza tra linee politiche teoriche e modalità pratiche venne avvertito e affrontato, complessiva-mente, con efficacia e coerenza.

Anzitutto, si trattò di operare una sorta di censimento: tutti i residenti stranieri vennero invitati a registrarsi presso le nuove autorità cittadine, facenti capo alla locale Commissione militare di controllo (军事管制委员会 junshi guanzhi weiyuanhui). Avrebbero dovuto rispettare gli orari del coprifuoco, non girare armati se non dietro rilascio di uno speciale permesso, consegnare eventuali apparecchi ricetrasmittenti in loro possesso, che sarebbero stati sigillati e restituiti nel momento in cui avrebbero lasciato la Cina ${ }^{15}$. A condizione che fossero in possesso di un passaporto regolare, osservassero leggi e ordinanze del nuovo governo e non svolgessero attività a esso ostili, sarebbero stati protetti.

Come accennato, la "nuova Cina" non avrebbe avuto fretta di instaurare nuove relazioni diplomatiche, a fronte di tutta una emergenza interna da gestire - e del rischio che accresciute presenze straniere complicassero, o sabotassero, l'andamento delle cose. Analogamente, anche dal punto di vista operativo, nei confronti dei "residenti stranieri" e di loro attività lecite o illecite in Cina, si procedette inizialmente per lo più con calcolata lentezza: per potere gestire la cosa nel modo migliore, e avendo tempo di acquisire anzitutto un quadro della situazione. Con l'eccezione di pochi membri che vi erano rimasti in condizio-

Beiping (北平, "pace del Nord”); alla fine del settembre 1949 i comunisti riportarono in uso la forma Beijing. In questa sede si userà la forma italianizzata.

14 È assai significativo in questo senso che tutti gli organismi previsti per il periodo di amministrazione militare avessero al loro interno una sezione o un responsabile preposti a seguire aspetti correlati alla politica estera e ai residenti stranieri: con ogni probabilità anche sulla scia del "caso Ward", console generale statunitense a Shenyang (沈阳): qui nel 1948 da parte delle autorità locali del PCC si erano verificate incongruenze di comportamento, e incomprensione di istruzioni delle autorità centrali del Partito, proprio in relazione al delicato ambito delle relazioni internazionali.

15 Bisogna naturalmente tenere presente che in quella fase di guerra civile il possesso di una radio ricetrasmittente aveva implicazioni particolari. 
ne di clandestinità, il Partito comunista riemergeva infatti a una dimensione urbana dopo una ventina d'anni di isolamento in contesti rurali, giungendo dall'esterno in ambienti da tutti i punti di vista lontani ed estranei alla grande maggioranza dei suoi uomini. Una delle priorità del periodo di amministrazione militare fu perciò di raccogliere informazioni sulla situazione e sulle varie realtà presenti nelle città, compito che avrebbe richiesto tempo. Pur tenendo presente il principio per cui non si doveva dare formale riconoscimento legale ai privilegi economici (经济特权 jingji tequan) del "capitale straniero", non si avrebbe dunque avuto fretta di far cessare le loro attività. Significative eccezioni a questo approccio attento e graduale furono le istituzioni finanziarie e le compagnie di navigazione fluviale straniere: dannose le prime per l' "economia del popolo", e lesive le seconde della sovranità nazionale, avrebbero dovuto fermare subito l'attività.

Coerentemente poi con la impostazione teorica per cui non si sarebbero riconosciute le relazioni diplomatiche allacciate dal governo nazionalista, il primo incontro con autorità e personale diplomatico straniero fu improntato a impersonalità e distanza, non esistendo ancora formali relazioni diplomatiche con alcun Paese. Non sarebbe stato loro riconosciuto alcun rango ufficiale, e sarebbero stati a tutti gli effetti considerati normali residenti stranieri, soggetti ai loro stessi obblighi e tutelati dalle stesse garanzie. Si sarebbe evitato di avere con loro qualsiasi intercorso, in un primo momento non comunicando loro neppure l'obbligo di presentarsi per la registrazione e il controllo del passaporto. Non esistendo relazioni ufficiali neppure con l'Unione Sovietica e gli Stati di "nuova democrazia", nei confronti del loro personale diplomatico si sarebbero avuti solo rapporti di tipo informale.

\section{LA “NUOVA CINA” E L'INTERVENTO IN COREA}

La guerra di Corea è un tema su cui la produzione storiografica è vastissima, e che ha presentato nel corso del tempo oscillazioni tra letture anche molto diverse, a partire dagli anni della guerra fredda e della immagine della "Stalin's War", fino a indagare le radici coreane del conflitto ${ }^{16}$. Nel periodo più recente, la disponibilità da un lato di nuove fonti cinesi, di studiosi cinesi formatisi negli Stati Uniti o cooperanti con progetti di ricerca americani, e dall'altro di materiale proveniente da archivi dell'ex Unione Sovietica, ha arricchito la

16 Una breve rassegna della storiografia sulla guerra di Corea è presentata in Solieri 1994, 129-34. Una vasta panoramica sui diversi temi e approcci è costituita da Brune, ed., 1996. Si segnala anche Shulman ed. 2014. 
storiografia sull'argomento con ricostruzioni estremamente dettagliate e minuziose, comunque non univoche ${ }^{17}$, e comunque quasi paralizzanti nelle loro rispettive esaustività.

L'enfasi sul post-Urss e sulle recenti fonti russe e cinesi ha poi contribuito a ricollocare in primo piano, curiosamente, una lettura del conflitto alla fine non lontanissima da quella degli anni della guerra fredda, ponendo al centro della scena Stalin e Mao Zedong. Vengono lasciati in penombra sia il contesto coreano sia la politica americana in Asia orientale e il clima negli Stati Uniti alla fine degli anni Quaranta-inizio degli anni Cinquanta, che pure ebbe un ruolo notevole, se non decisivo, nella escalation del conflitto: da operazione volta a respingere le truppe nordcoreane al di là del $38^{\circ}$ parallelo a missione mirante a eliminare il governo di Pyongyang ${ }^{18}$.

In questa sede si cercherà naturalmente di svolgere, relativamente alle prime risposte cinesi alla evoluzione della situazione in Corea, solo qualche considerazione sintetica e mirata. In particolare, verranno lasciate sullo sfondo la complessità del processo decisionale che allora ebbe luogo, e anche la situazione interna: una decurtazione importante, alla luce anche delle interconnessioni tra situazione internazionale e scenario domestico ${ }^{19}$.

Due giorni dopo lo scoppio del conflitto coreano, il 27 giugno, su proposta statunitense il Consiglio di sicurezza dell'ONU deliberò, a fronte del rifiuto nordcoreano di ritirarsi al di là del $38^{\circ}$ parallelo, che gli Stati membri fornissero alla Corea del Sud "tutta l'assistenza necessaria". Il delegato sovietico Malik, continuando la protesta di Mosca per la mancata ammissione all'ONU della Repubblica Popolare Cinese, non partecipò alla seduta, come non aveva partecipato a quella del 25 giugno: non fece quindi uso del proprio diritto di veto. La circostanza è stata oggetto di molte speculazioni, alla luce delle conseguen$z e$ in termini di isolamento internazionale e di embargo commerciale ${ }^{20}$ che il coinvolgimento nel conflitto in Corea ebbe per Pechino. Dal punto di vista della Repubblica Popolare Cinese, a suscitare in un primo momento preoccupazione fu la decisione statunitense, sempre il 27 giugno, di spostare la Settima flotta a pattugliare lo stretto di Taiwan, allo scopo di impedire che venisse con-

17 Ad esempio, Heinzig e Chen Jian danno letture assai diverse del soggiorno moscovita di Mao Zedong, nell'inverno 1949-1950, privilegiando l'uno le fonti ex- sovietiche e l'altro le recenti fonti cinesi.

18 È questa la impostazione dei Sino-Soviet Studies, che hanno prodotto studi importanti, ma all'interno di una visione inevitabilmente monca del quadro d'insieme: si veda al riguardo: Solieri 2003/2004, 197-200). Recentemente, la tendenza a ridurre la prospettiva quasi esclusivamente a Urss e RPC, lasciando sullo sfondo la politica americana in Asia orientale e nel mondo, è molto viva: cfr. Lüthi 2008 e Shen 2012.

19 Si vedano le considerazioni sui rapporti tra guowai (国外 esterno del Paese) e guonei (国内 interno del Paese) in Solieri 2006, 177-204.

20 L'embargo venne introdotto dal dicembre 1950 da parte degli Stati Uniti, e dal 1951 dai Paesi alleati, con modalità e tempistiche che seguiranno parabole diverse. 
quistata da "forze comuniste": agli occhi di Truman era infatti evidente che il "Comunismo" da quel momento in poi sarebbe passato a invasione armata e guerra per conquistare Paesi indipendenti. Dal punto di vista di Pechino, con la mossa americana venne a crearsi una situazione doppiamente grave: pericolosa da un punto di vista obiettivo, e in radicale contrapposizione col principio di non interferenza straniera negli affari interni della "nuova Cina" ${ }^{21}$.

Da parte statunitense continuarono poi a giungere segnali contraddittori, da parte di figure diverse, che non permettevano di mettere a fuoco né una chiara linea politica né un chiaro referente: in particolare, in luglio ci fu una visita con dichiarazione congiunta del generale MacArthur ${ }^{22}$ a Chiang Kai-shek, a Taiwan, all'insaputa del Dipartimento di Stato: rafforzò in Cina il sospetto di un attivo coinvolgimento del Guomindang nelle decisioni americane e rinfocolò la opposizione anticomunista interna. L'iniziativa fu solo la prima di una serie di mosse e dichiarazioni analoghe, che il generale avrebbe fatto senza consultarsi con Washington. In agosto, iniziando l'andamento dei combattimenti a pendere dalla parte del corpo di spedizione internazionale e rientrato in seno al Consiglio di sicurezza il rappresentante sovietico, per assumerne a rotazione la presidenza, il delegato statunitense iniziò a sostenere che scopo dell'intervento ONU in Corea non era ripristinare lo status quo, ma continuare i combattimenti fino alla eliminazione del governo di Pyongyang e alla unificazione della intera penisola (Gurtov, Hwang 1980, 50).

La prospettiva di truppe statunitensi ai confini con le province nordorientali, cuore strategico dell'industria pesante cinese e della ricostruzione, fece sì che un telegramma di Zhou Enlai del 20 agosto al Consiglio di sicurezza focalizzasse per la prima volta sulla Corea e non su Taiwan la attenzione del governo cinese, chiedendo tra l'altro di partecipare alla discussione in sede di Consiglio e il ritiro di tutte le truppe straniere dalla penisola. Le richieste cinesi vennero respinte, e da fine agosto bombardieri americani effettuarono bombardamenti di installazioni ferroviarie e popolazione civile sul suolo cinese, a ridosso del confine con la penisola coreana ${ }^{23}$.

Fattori interni americani quali le imminenti elezioni del Congresso, l'euforia dopo i successi militari e il riuscito sbarco ONU a Inchon, unitamente a considerazioni di strategia globale, fecero sì che in settembre maturassero negli

${ }_{21}$ Significativamente, un telegramma del 6 luglio al Segretario generale dell'ONU affermava che la risoluzione e le decisioni di Truman del 27 giugno costituivano atti di aperta aggressione e intervento negli affari interni di Corea e Repubblica Popolare Cinese. In effetti, in Corea era in atto una guerra civile che aveva già causato centomila morti; fino alla prima guerra contro l'Irak, l'intervento ONU in Corea, direttamente a sostegno di una delle parti in causa anziché mirante a frapporvi un corpo di pace, è stato un fatto eccezionale.

22 Allora comandante del corpo di intervento internazionale in Corea.

23 Già a luglio MacArthur si era detto convinto dell'utilità di bombardare ai confini della Cina allo scopo di prevenire un intervento sovietico o cinese. 
Stati Uniti le condizioni per cui lo stesso Truman iniziò a parlare di una Corea "libera, indipendente e unita". La situazione evolvette dunque nella direzione di un secondo mandato ONU, che modificasse e ampliasse l'obiettivo originario, mirando a eliminare lo stesso governo nordcoreano. A quel punto, di fronte alla prospettiva di truppe americane ai confini di Russia e Cina, tra Mosca e Pechino si raggiunse un accordo di massima che prevedeva un intervento di terra cinese con copertura aerea russa (Hong 1991, 25) ${ }^{24}$. Pechino, pur curando per tempo l'aspetto dei preparativi militari, tentò fino in fondo la carta delle trattative diplomatiche, entro i limiti di ascolto e visibilità internazionali di cui allora disponeva. Cercò di venire informata attraverso l'India dei colloqui che si svolgevano in ambito internazionale e tentò di fare arrivare agli Stati Uniti, attraverso l'ambasciatore indiano a Pechino, Panikkar, il proprio senso della gravità della situazione.

Il 25 settembre quest'ultimo ebbe a Pechino un colloquio col generale Nie Rongzhen (聂荣臻), comandante della regione militare di Pechino-Tianjin e Capo di Stato maggiore sotto Zhu De (朱德): Nie affermò che la aggressione americana avrebbe dovuto essere fermata a ogni costo, anche se gli Stati Uniti avessero usato la bomba atomica contro la Cina.

Un secondo avvertimento, a un livello ancora più alto, venne mandato il 2 ottobre, dopo che Pechino aveva avuto notizia del fatto che, mescolati a truppe coreane, anche soldati statunitensi avevano varcato di nascosto il $38^{\circ}$ parallelo, e stavano avanzando verso i confini cinesi e la vasta area nordorientale, che era il centro nevralgico della industria pesante cinese e di importanza cruciale per la ricostruzione. Anche se gli Stati Uniti non avessero attaccato direttamente la Repubblica popolare, avrebbero potuto stabilire un governo ostile in Corea ed esercitare lungo tutto quel delicato confine un'intollerabile pressione militare, continuando allo stesso tempo a fornire attivo supporto ai nazionalisti del Gmd, che miravano a tornare al potere sulla terraferma. L'impresa di sostenere una difesa passiva della regione era impossibile:

Quante truppe sono necessarie per sorvegliare il fiume Yalu, lungo un migliaio di chilometri? Inoltre, dovremmo aspettare là anno dopo anno senza sapere quando il nemico arriverà. (Zhou 1984, 2: 52)

Si sarebbe quindi determinata una congiuntura, dal punto di vista della dirigenza cinese, suscettibile di costituire a tempo indefinito una minaccia lungo un confine di massima importanza strategica, e di saldare minaccia esterna e rischio di destabilizzazione interna.

Il 2 ottobre Mao Zedong informò quindi Stalin della decisione cinese di

24 Il timore di Stalin era che l'Urss, se si fosse esposta in prima persona a fianco della Corea del Nord, potesse venire attaccata anche sul fronte occidentale, in Europa, causando una terza guerra mondiale. 
mandare un corpo di “volontari del popolo cinese" (中国人民志愿军 Zhongguo renmin zhiyuanjun) ${ }^{25}$ a combattere a fianco dei nordcoreani, perché "se la Corea viene occupata dagli Americani le forze rivoluzionarie coreane sarebbero completamente eliminate e gli imperialisti americani diventerebbero ancora più aggressivi, il che sarebbe un male per l'intero Oriente" (Hong 1991, 25).

Contemporaneamente, venne mandato un ultimo avvertimento per via diplomatica, nelle intenzioni dei cinesi massimamente chiaro e autorevole: l'ambasciatore indiano venne condotto, in piena notte, a un colloquio con Zhou Enlai, in cui venne chiarito quello che per la Repubblica popolare rappresentava il limite da non superare, il casus belli: se truppe americane - nulla venne detto circa quelle sudcoreane - avessero passato il $38^{\circ}$ parallelo, la Cina non avrebbe potuto non intervenire (Panikkar 1955, 109-10). Ma, anche in questo caso, l'avvertimento restò ignorato ${ }^{26}$ : il 7 ottobre, lo stesso giorno in cui l'Assemblea generale delle Nazioni unite - in sede di Consiglio di sicurezza l'Unione Sovietica avrebbe potuto fare uso del proprio diritto di veto - approvava il nuovo mandato della spedizione internazionale, anche truppe statunitensi passarono in forze nella Corea del Nord.

Al termine di un processo decisionale di non sovrastimabili gravità e complessità, aggravato dal ritiro sovietico della iniziale disponibilità a fornire da subito copertura aerea, la RPC inviò dunque un contingente di "volontari" a combattere in Corea: la decisione è ritenuta una delle più difficili che Mao Zedong, cui viene in sintesi riconosciuta la convinzione che l'intervento non fosse evitabile, abbia mai dovuto prendere. Diciotto divisioni filtrarono dal 19 ottobre attraverso il confine, di notte e inosservate.

Interessantemente, però, anche allora la Repubblica popolare non rinunciò a una mossa che lasciasse spazio di riflessione: dopo un primo e intenso scontro, per tre settimane si perse ogni contatto con le sue truppe: appare convincente l'ipotesi che vede in quella pausa una mossa interlocutoria, volta a lasciare aperta una via d'uscita negoziale, dopo aver chiarito agli occidentali che gli avvertimenti non erano stati un bluff (Whiting 1960, 42). Da parte cinese poteva trattarsi di una dimostrazione di determinazione ad agire e allo stesso tempo di disponibilità a evitare, se possibile, un conflitto. Ma a Washington, dove nelle elezioni del 7 novembre i repubblicani avevano aumentato la propria rappresentanza al Congresso attaccando da posizioni maccartiste la politica di Truman e Acheson in Asia, non c'era posto per dimostrazioni di

25 La denominazione "volontari del popolo cinese" venne scelta per ridurre il senso di una contrapposizione frontale con gli Stati Uniti.

26 Complessivamente, a Washington prevalse la convinzione che Pechino, a fronte della gravità dei compiti sul fronte interno, della prudenza di Mosca, e anche del fatto che un intervento avrebbe grandemente accresciuto la sua dipendenza dall'Unione Sovietica, avrebbe evitato di mandare uomini in Corea. 
'debolezza': della condotta cinese sul campo si diede una lettura che la vedeva dettata da indecisione e mancanza di preparazione militare.

Senza affrontare qui ulteriori aspetti e vicende legate all'intervento cinese in Corea - ma a parere di chi scrive sarebbe più corretto parlare di coinvolgimento militare - emerge anche da questi brevi e limitati cenni qualche spunto di riflessione.

Emerge anzitutto sotto quale complessa costellazione - a cavallo tra decolonizzazione e guerra fredda ${ }^{27}$ - sia avvenuta la imprevista nascita della "nuova Cina". Sia Usa che Urss avevano cercato, dal 1945, di evitare un intervento diretto in Cina, conservandovi però un certo grado di influenza sulla situazione interna; la Repubblica popolare, la cui intenzione di chiudere con l'"imperialismo" straniero era radicale, si trovò a fronteggiare da parte statunitense una percezione monolitica e monoliticamente minacciosa del comunismo mondiale, e da parte sovietica diffidenza e timori.

Lo stato attuale della ricerca evidenzia come linee politiche e decisioni di fondo relative alla politica estera siano state allora impostate e prese autonomamente dai dirigenti del PCC e poi anche della RPC: perfino la cruciale decisione di inviare un corpo di spedizione a combattere a fianco della Corea del Nord. Intervistato da chi scrive, un ufficiale dell'Epl che ha avuto accesso privilegiato a materiale d'archivio e che sulla guerra di Corea ha scritto un'opera dal notevole valore storico e documentario (Ye 1989) ha sostenuto con decisione che l'Unione Sovietica non era in condizione di imporre niente alla Repubblica Popolare Cinese: affermazione che, in considerazione del contorto percorso storico tra Unione Sovietica e PCC della prima metà del Novecento, appare, nella sua concisione, plausibile.

$\mathrm{Si}$ è anche visto come, in diverse circostanze e relativamente ad aspetti diversi, il PCC abbia cercato di veicolare a Washington segnali che non si seppe, o volle, vedere o interpretare. Non è questa la sede per approfondire aspetti legati al clima della guerra fredda negli Stati Uniti, o all'influenza delle scadenze elettorali statunitensi sulle decisioni di politica estera, o al ruolo dei massmedia nell'orientarvi l'opinione pubblica; tuttavia, la mancata comprensione degli avvertimenti lanciati dalla Cina a fronte dell'escalation in Corea merita qualche riga di approfondimento. I segnali mandati da Pechino vennero infatti commentati pubblicamente in modo che suona inverosimile: sarebbero stati "un bluff per influenzare il voto alle Nazioni unite" (Tang 1963, 573-5); Pechino avrebbe dovuto alzare la posta se voleva entrare in gioco; Zhou Enlai non sarebbe stato un interlocutore politico autorevole. Alla fine, gli avvertimenti vennero ignorati anche perché giungevano sgraditi, quando cioè chi doveva decidere aveva già deciso: prenderli sul serio avrebbe significato mettersi in

27 È questa la impostazione di Osterhammel 1992. 
contrapposizione a MacArthur, rischiare nuove accuse di arrendevolezza, perdere consensi elettorali.

$\grave{E}$ allo stesso tempo anche vero che gli Stati Uniti ebbero allora realmente difficoltà a mettere a fuoco la portata della rottura col passato che i comunisti cinesi intendevano operare nel campo delle relazioni internazionali: a esempio, un memorandum del Joint Intelligence Committee dei primi di luglio 1950, che elencava le ragioni per cui un intervento cinese pareva da escludersi, metteva al primo posto ragioni legate alla ricostruzione economica, pena la tenuta del nuovo governo. Del tutto antitetica, invece, la impostazione delle valutazioni che il 27 settembre il generale Nie Rongzhen espresse all'ambasciatore indiano Panikkar:

Sappiamo quel che ci aspetta, ma la aggressione americana deve essere fermata a qualunque costo. Gli Americani possono bombardarci, possono distruggere le nostre industrie, ma non possono sconfiggerci sul campo. Possono anche lanciare delle bombe atomiche. E poi? Possono ammazzare qualche milione di persone. L'indipendenza di una nazione non può essere salvata senza sacrifici. Dopo tutto, la Cina vive della sua agricoltura. Cosa possono quindi farci le bombe atomiche? Sì, il nostro sviluppo economico sarebbe ritardato. Possiamo aspettarcelo. (Panikkar 1955, 108)

Dal punto di vista di Pechino, quindi, a essere prioritaria era la difesa della indipendenza nazionale. Accanto alla difesa di quest'ultima, furono anche la mancanza di risposte e la invisibilità internazionale che avvolgevano la "nuova Cina" a risultare insostenibili. In questo senso, l'intervento cinese in Corea "served the function of clarifying China's position in the world"(Adelman, Shiy 1993, 191), facendo chiarezza anche sulle nuove priorità della "nuova Cina": Stato che nasceva da una rivoluzione, e la cui fondazione costituiva, e doveva rendere visibile, "the emergence of a revolutionary power" 28.

Da parte statunitense, a risultare in ultima analisi inaccettabile fu proprio la nascita di un governo rivoluzionario, programmaticamente intenzionato a rompere con la propria eredità in campo diplomatico:

For Acheson the Chinese Communists' attitude toward their "international obligations", which included treaties and debts of the KMT [Gmd] government, showed weather they would be an "evolutionary regime" or a "revolutionary one". The Unites States, Acheson told the British Ambassador to the United States, would do all it could do to prevent the latter development. The contention over international obligations, in fact, was really about revolution and counterrevolution. American diplomatic personnel gradually left the mainland, not to return for thirty years. (Chang 1990,33)

\footnotetext{
28 Così titola Chen Jian l'intera prima parte della sua analisi (Chen 1994, 9-30).
} 


\section{LA POLITICA ESTERA DELLA “NUOVA CINA”: CENNI SU ALCUNE COORDINATE STORICHE}

Poco dopo la fondazione della Repubblica Popolare Cinese, nell'aprile 1952, in occasione di un incontro con gli ambasciatori stranieri presenti in Cina, Zhou Enlai sintetizzò linee e compiti di politica estera, anzi della "politica estera di pace". Si tratta, come si vedrà, di alcuni grandi principi già emersi nelle fasi e circostanze sintetizzate in questo contributo, o comunque coerenti con orientamenti di fondo allora già presenti:

1. “Mettere su un nuovo focolare" (另起炉灶 lingqi luzao);

2. "Pendere da una parte" (一边倒 yibiandao);

3. “Invitare ospiti solo dopo aver fatto pulizia in casa” (打扫干净屋子再请客 dasao ganjing wuzi zai qing ke);

4. “La cortesia/etichetta/rito richiede reciprocità"(礼尚往来 li shang wanglai): nei confronti dei Paesi capitalisti, se questi si fossero comportati bene con la Cina, la Cina si sarebbe comportata bene con loro; se non si fossero comportati bene, sarebbero stati ripagati con la loro stessa moneta;

5. "Scambiare quello che si ha con quello di cui si ha bisogno" (互通有无 bu tong youwu): la Repubblica Popolare Cinese avrebbe portato avanti rapporti di scambio commerciale con Paesi stranieri sulla base di uguaglianza e vantaggio reciproco;

6. “Unirsi ai popoli del mondo" (团结世界人民 tuanjie shijie renmin): la Cina "pendeva dalla parte" dell'Unione Sovietica e delle democrazie popolari, ma non avrebbe rinunciato a unirsi a, e a persuadere, anche i popoli dei Paesi capitalisti e imperialisti (Zhou 1994, 2: 85-8).

È molto interessante osservare che di lì a un paio di anni queste grandi linee di fondo sarebbero confluite nei "cinque principi di coesistenza pacifica", che a tutt'oggi costituiscono il nucleo portante della politica estera cinese ${ }^{29}$.

Volendo tentare di ampliare la prospettiva, cercando di sintetizzare brevemente qualche considerazione sull'orizzonte storico-culturale da cui tali grandi orientamenti di fondo sono nati, non si può anzitutto non ricordare la ricca pluralità di modalità con cui la Cina imperiale si rapportava al mondo circostante. Alcune disposizioni culturali, politiche e strategiche storicamente ricorrenti possono, muovendo da tale prospettiva temporale estesa, essere identificate in:

29 I cinque principi sono: rispetto reciproco per sovranità e integrità territoriali; reciproca non-aggressione; non interferenza negli affari interni altri Paesi; uguaglianza e vantaggio reciproco; coesistenza pacifica. Per la loro importanza alla Conferenza di Bandung del 1955 e in relazione in particolare alla attuale politica africana della Cina, cfr. Samarani 2010, 21-23. Su come la loro centralità sia stata recentissimamente ricordata anche agli Stati Uniti, cfr. Pieranni 2014, 7. 
1. una forte preoccupazione per la sicurezza territoriale delle zone periferiche della Cina;

2. la consapevolezza dell'importanza di preservare uno Stato unitario a fronte del pericolo di ricadere nel disordine del periodo degli Stati combattenti (战国 zhanguo 453-222 a.C.);

3. la esperienza storica della debolezza politica della Cina del periodo tardoQing (清1644-1911) e del conseguente bisogno di una diplomazia difensiva, nonché nel senso di umiliazione a fronte delle potenze occidentali e del Giappone;

4. la ambivalenza tra indipendenza e dipendenza sperimentata a partire dalle Guerre dell'Oppio e anche, nel XX secolo, nella tensione tra nazionalismo e internazionalismo.

Nel XX secolo la tensione tra nazionalismo e internazionalismo fu, si potrebbe dire, connaturata al Partito Comunista Cinese fin dalla sua nascita, favorita da un intervento del Comintern.

A partire dal 1927 (Samarani 2004, 83-4) il PCC sperimentò poi nel modo più doloroso, sulla propria pelle, la complessità di declinare una prospettiva "China centered" con una prospettiva facente capo ad attori esterni. Alla traumatica eliminazione fisica dei comunisti dalla scena urbana di quell'anno concorsero infatti fattori tra loro del tutto antinomici, ma entrambi riconducibili in ultima analisi ad attori stranieri: da un lato le pressioni da parte sovietica perché il Partito comunista si alleasse con quello nazionalista; dall'altro gli interessi finanziari e commerciali occidentali in Cina, cui Chiang Kai-shek scelse di guardare e compiacere. Anche dal punto di vista delle esperienze personali della generazione cui appartenevano i fondatori del PCC, i fatti dell'aprile del 1927 e del periodo successivo costituirono la più traumatica delle esperienze, e posero in termini prioritari e assoluti, senza che alternative fossero possibili, il problema della sopravvivenza: personale e politica (Zhang 1987, passim).

Un atteggiamento difensivo nei confronti di forti Paesi interventisti ha le sue origini dunque non in una presa di posizione astratta, ma nel bagaglio di esperienze, nella costellazione di vicende politiche e personali vissute dal partito e dai suoi membri: una fondamentale lezione del passato, non un tatticismo.

Ai tratti di fondo del quadro da cui si sviluppò l'approccio dei comunisti cinesi alle relazioni estere, non si può poi non aggiungere, accanto al ricordato vivo senso di umiliazione nei confronti dei Paesi "imperialisti", un forte senso dello Stato ${ }^{30}$. Fu il senso dello Stato che accentuò la gravità della minaccia rappresentata da un dominio straniero. La generazione dei fondatori del PCC era stata testimone della prolungata crisi tardo-Qing e dei primi tentativi repubblicani: loro e altri intellettuali politicamente impegnati dimostrarono una

30 Sulla espressione resa con “costruzione dello Stato" (建国 jianguo) si veda Solieri 2006, 12. 
così marcata sensibilità per la salvezza dello Stato e una così notevole volontà di dedicare le proprie vite al suo recupero da sembrarne, visti dall'esterno, come "ossessionati", portatori di una tutta cinese "ossessione per lo Stato" (Schwartz 1987, 1-10). Si è a esempio brevemente notato, anche nella stringatezza e nei limiti di questo contributo, come nei primissimi mesi del 1949, quando la proclamazione della Repubblica Popolare Cinese era ancora lontana, le più alte autorità del Partito riflettessero sulle future relazioni internazionali della "nuova Cina" in termini già per così dire proto-statuali. A sua volta, è evidente in prospettiva storica come il rimodellamento del nuovo Stato cinese abbia comportato nei fatti anche un rimodellamento dello scenario internazionale.

Si è già accennato alla complessità del processo decisionale che portò all'intervento cinese in Corea. In particolare, volendo esemplificare grandemente, nei primi diciassette giorni di ottobre, quando pure gli eventi incalzavano e il tempo era prezioso, tra Mao Zedong e i più alti responsabili del partito e dell'Esercito popolare di liberazione si tennero complesse, ripetute, esaustive consultazioni. Anche questo aspetto, cioè la preferenza da parte di Mao per fare emergere una linea condivisa, attraverso mediazione politica e ampie discussioni, su importanti aspetti collegati alla politica estera, è espressione di un orientamento rintracciabile dai suoi primi anni alla guida del PCC (Hunt n. d., 180-1).

Con lo scoppio della guerra di Corea, di fronte alla prospettiva che il conflitto si allargasse sul suolo cinese e che americani e nazionalisti 'spazzassero via' i comunisti, i nemici interni del nuovo governo videro rafforzarsi le proprie speranze. Gruppi di oppositori, cui allora si unirono proprietari terrieri, società segrete, soldati del Gmd sbandati, intensificarono le loro attività. Portarono avanti in tutto il Paese atti di violenza e intimidazione, colpendo anche nel Nordest, la base logistica che sosteneva le operazioni militari in Corea. Fecero circolare voci circa l'imminenza della terza guerra mondiale e cercarono di esercitare pressione sui quadri locali del PCC. Pechino, si potrebbe dire, concordava con i suoi nemici interni che il conflitto coreano potesse estendersi alla Cina e unire gruppi dissidenti interni con una forza di invasione americanonazionalista. Dal 10 ottobre il Comitato centrale del partito si rivolse ai controrivoluzionari con una determinazione maggiore, ponendo fine a un approccio che era stato fino ad allora troppo morbido nei confronti dei nemici del nuovo governo (Solieri 2006, 198-203). La fiammata di resistenza contro-rivoluzionaria che seguì lo scoppio del conflitto coreano fu uno dei diversi elementi che contribuirono, di lì a un paio di anni, a introdurre una accelerazione verso la transizione al socialismo. 


\section{BIBLIOGRAFIA PRIMARIA}

Bo, Yibo. 1991. Ruogan zhongda juece yu shijian de buigu (Retrospettiva di importanti decisioni politiche e avvenimenti). Beijing: Zhonggong Zhongyang dangxiao chubanshe, vol. 1.

Hong, Xuezhi. 1991. KangMei yuanChao zhanzheng buiyi (Ricordi della guerra per resistere alla aggressione statunitense e sostenere la Corea). Beijing: Jiefangjun wenyi chubanshe.

Lie, Trigve. 1954. In the cause of Peace: Seven Years within the United Nations. New York: Macmillan.

National Archives. 1945-1949. "Approaches by Chinese Communists Regarding Economic Relations with the U.S." College Park, MD. Confidential U.S. State Department Central Files-China-Internal Affairs. RG 59, LM 185, roll 5.

Panikkar, Kavalam Madhava. 1955. In Two Chinas. London: Allen and Undwin.

Shi Zhe. 1991. Zai lishi juren shenbian (A fianco di giganti della Storia). Beijing: Zhongyang wenxian chubanshe.

van Slyke, L. P. ed. 1967. The China White Paper. Stanford: Stanford University Press.

ZhongGong Zhongyang wenxian yanjiushi bian. 1993. Mao Zedong nianpu (Cronaca della vita di Mao Zedong). Beijing: Zhongyang wenxian chubanshe-Renmin chubanshe, vol. 3 .

Zhongyang dang' anguan bian. 1992. ZhongGong Zhongyang wenjian xuanji (Selezione di documenti del Comitato Centrale del Partito Comunista Cinese). Beijing: ZhongGong Zhongyang dangxiao chubanshe, vol. 17.

Zhongyang dang' anguan bian. 1992. ZhongGong Zhongyang wenjian xuanji (Selezione di documenti del Comitato Centrale del Partito Comunista Cinese). Beijing: ZhongGong Zhongyang dangxiao chubanshe, vol. 18.

Zhou, Enlai. 1984. Zhou Enlai xuanji (Opere scelte di Zhou Enlai). Beijing: Renmin chubanshe, vol. 2.

\section{BIBLIOGRAFIA SECONDARIA}

Adelman, J.R., C. Shih. 1993. Symbolic War: The Chinese Use of Force, 1840-1980. Taipei: National Chengchi University.

Brune, L.H., ed.1996. The Korean War. A Handbook of the Literature and Research. Westport: Greenwood Press.

Chang, G.H. 1990. Friends and Enemies. The United States, China and the Soviet Union, 1948-1972. Stanford: Stanford University Press. 
Chen, J. 1994. China's Road to the Korean War. The Making of the Sino-American Confrontation. New York: Columbia University Press.

Gurtov, M., B.M. Hwang. 1980. China under Threat. The Politics of Strategy and Diplomacy. Baltimore: The Johns Hopkins University Press.

Heinzig, D. 1998. Die Sowjetunion und das kommunistische China 1945-1950. Der beschwerliche Weg zum Bündnis. Baden Baden: Nomos Verlagsgesellschaft.

Hunt, M.H. n. d. “Ccp Foreign Policy: 'Normalizing' the Field”, in Hunt, Niu (eds, n. d.), 163-91.

Hunt, M.H., J. Niu, eds, n. d. Toward a History of Chinese Communist Foreign Relations, 1920s-1960s. Washington: Woodrow Wilson International Center.

Lüthi, L.M. 2008. The Sino-Soviet Split: Cold War and the Communist World. Princeton: Princeton University Press.

Medvedev, R. 1986. China and the Superpowers. Oxford: Basil Blackwell.

Osterhammel, J. 1992. Storia della Cina moderna (secoli XVIII-XX). Torino: Einaudi.

Pieranni, S. 2014. "Pechino e Washington sempre più compromessi." il manifesto, 10. 7: p.7.

Samarani, G. 2004. La Cina del Novecento. Dalla fine dell'Impero a oggi. Torino: Einaudi.

Samarani, G. 2010. Cina, ventunesimo secolo. Torino: Einaudi.

Schramm S., ed. 1987. Foundations and Limits of State Power in China. London: School of Oriental and African Studies.

Schwartz, B. I. 1987. “The Primacy of the Political Order in East Asian Societies: Some Preliminary Generalizations.” In Schramm ed. 1987: 1-10.

Shaw, Y. 1982. "Communist Rapprochement in 1949: Was There Another 'Lost Chance' in China?" The China Quarterly 89: 76-96.

Shen, Z. 2012. Mao, Stalin and the Korean War: Trilateral Communist Relations in the 1950's. London: Routledge.

Shulman, F.J., ed. 2014. A Century of Doctoral Dissertations on Korea, 1903-2004: An Annotated Bibliography of Studies in Western Languages. Brill: Leiden, forthcoming.

Simmons, R. 1975. The Strained Alliance. Peking, P'yongyang, Moscow and the Politics of the Korean Civil War. New York: The Free Press.

Solieri F. 1994. "Le molte guerre di Corea." Passato e Presente 32: 129-34.

Solieri F. 2003/2004. "Recensione di Dieter Heinzig, The Soviet Union and Communist China 1945-1950: The Arduous Road to the Alliance." Asiatica Venetiana 8/9: 197-200.

Solieri F. 2006. Cina 1948-1950. Il Partito Comunista Cinese e il passaggio dalla Guerra civile alla costruzione dello Stato. Milano: FrancoAngeli.

Stueck, W.W. 1981. The Road to Confrontation: American Policy Toward China and Korea 1947-1950. Chapel Hill: University of North Carolina Press. 
Tang T. 1963. America's Failure in China 1941-1950. Chicago: University of Chicago Press.

Whiting, A.S. 1960. China Crosses the Yalu. The Decision to Enter the Korean War. Stanford: Stanford University Press.

Ye, Y. 1989. Hei xue (Neve nera). Beijing: Zuojia chubanshe.

Zhang, Z. 1987. Zhongguo xiandai geming shi shiliaoxue (Storiografia sulla moderna storia rivoluzionaria cinese). Beijing: ZhongGong dangshi ziliao chubanshe.

\section{ABSTRACT}

When victory in the civil war (1946-49) seemed near, the Chinese Communist Party defined a first series of important directives for the future foreign policy of the People's Republic. This paper considers such directives in the tense international context which accompanied the creation of the new state, delineating in particular the complex web of relations between Chinese Communists, the Soviet Union, the United States of America, and the United Nations. During the years of the Cold War a strongly polarised world-view prevented the identification of the specific historical and even cultural choices that the Chinese made at the time; this essay tries to reconstruct these, identifying, on the one hand, roots that lie in the past, and, on the other, their continued relevance for China's foreign policy today. This essay is based, amongst other things, on the most recent and authoritative Chinese historical sources, archival sources on the US, and on historiography in both Western languages and in Chinese. 